\title{
Organerhaltung, multimodale radikale Tumortherapie und Reduktion der therapiebedingten Morbidität
}

\author{
Entwicklung in Diagnostik und Therapie des Endometriumkarzinoms
}

\author{
R. Kimmig, Essen D. Fink, Zürich U. Lang, Graz
}

\begin{abstract}
Zusammenfassung
Das Endometriumkarzinom ist das häufigste Genitalmalignom der Frau. Eine individualisierte Therapiestrategie ist angesichts der tumorbiologisch und prognostisch sehr unterschiedlichen Subtypen des Endometriumkarzinoms von hoher Bedeutung. Neben der Frage des individualisierten organerhaltenden Vorgehens bei Frauen mit Kinderwunsch wird die Reduktion der Morbidität durch minimalinvasive laparoskopisch/vaginale Chirurgie diskutiert. Während die adjuvante Gestagentherapie keinen Nutzen bringt, scheint eine platinhaltige Chemotherapie in bestimmten Risikosituationen wirksam zu sein. Die Indikation zur Lymphonodektomie in Bezug auf das Staging sowie die Wirkung der adjuvanten Strahlentherapie zur lokalen Kontrolle sind unumstritten. Im Hinblick auf einen postulierten kurativen Effekt werden uns die Ergebnisse der laufenden prospektiven Studien weitere Klarheit bringen.
\end{abstract}

\section{Organ Preservation, Multimodal Radical Tumor Therapy and Reduction of Therapy-Related Morbidity - Development of Diagnostics and Therapy of Endometrial Carcinoma}

Endometrial carcinoma is the most frequent genital malignoma in women. Considering the tumor-biological and prognostically very different subtypes of endometrial carcinoma, an individual therapeutic strategy plays a very important role. Besides the question of an individualized organ-preserving procedure for women who wish to have a baby, the reduction of morbidity by minimally invasive laparoscopic/vaginal surgery is discussed. While adjuvant gestagen therapy is not useful, platinum-containing chemotherapy seems to be effective in certain risk situations. The indication of lymphonodectomy with regard to staging as well as the effect of adjuvant radiation therapy for local control are undisputed. In view of a postulated curative effect, the results of the ongoing prospective studies will make things clearer.
Das Endometriumkarzinom ist in Europa und Nordamerika das häufigste Genitalmalignom der Frau, wobei geschätzt allein in Europa etwa 9000 Frauen im Jahr an dieser Erkrankung sterben [1].

Es gilt als Tumorerkrankung mit prinzipiell guter Prognose, da die relative 5-Jahres-Überlebensrate etwa 80\% beträgt; hinsichtlich der prognostischen Einschätzung sind jedoch tumorbiologisch zwei unterschiedliche Subtypen des Endometriumkarzinoms zu unterscheiden: zum einen das typische, hormonsensitive Endometriumkarzinom mit guter Differenzierung und in der Regel geringer Infiltrationstiefe mit sehr guter Prognose (Typ 1) sowie andererseits hormonunabhängige und schlecht differenzierte Tumore. Diese zeigen oft frühzeitig bereits eine lymphatische Invasion und besitzen eine deutlich erhöhte Metastasierungstendenz (Typ 2). Es ist daher nahe liegend, die therapeutische Strategie dem individuellen Risiko anzupassen, zumal Frauen mit Endometriumkarzinom häufig mehrere Komorbiditäten aufweisen, die das perioperative Risiko deutlich erhöhen können.

Unwidersprochen ist die primär operative Therapie die Therapie der Wahl. Die retroperitoneale Lymphadenektomie ist im Rahmen des Stagings unumstritten, wohingegen eine kontroverse Diskussion hinsichtlich des kurativen Charakters und des notwendigen Ausmasses der retroperitonealen Lymphadenektomie besteht. Dies gilt auch für die Frage der adjuvanten Bestrahlung, wo mehrere grosse Studien eine verbesserte lokale Kontrolle, jedoch keine Verbesserung des Überlebens zeigen konnten [2]. Mit Spannung erwarten wir diesbezüglich die Ergebnisse der PORTEC-2-Studie, des NCICEN-5-Trials sowie des bezüglich Patientinnenzahl grössten ASTECTrials.

\begin{tabular}{ll}
\hline KARGER & ( 2006 S. Karger AG, Basel \\
Fax +4161306 1234 & \\
$\begin{array}{l}\text { E-Mail karger@karger.ch } \\
\text { www.karger.com }\end{array}$ & $\begin{array}{l}\text { Accessible online at: } \\
\text { www.karger.com/ggr }\end{array}$
\end{tabular}


Im Rahmen der Primärtherapie erbringt eine adjuvante Gestagentherapie keinen Vorteil und sollte daher nicht durchgeführt werden [3]. Ein hochinteressanter Ansatz ist jedoch die Gestagentherapie von Frühstadien des Endometriumkarzinoms mit dem Ziel der Organerhaltung bei jungen Frauen mit Kinderwunsch. In einem umfassenden Überblick werden die hierzu vorliegenden Daten analysiert. In individuellen Fällen nach sorgfältiger Nutzen-/Risiko-Abwägung scheinen eine Organerhaltung und konsekutiv die Erfüllung des Kinderwunsches möglich.

Bei der Behandlung des Hochrisikoendometriumkarzinoms finden sich zunehmend Hinweise auf eine Wirksamkeit der Chemotherapie in der adjuvanten Situation. Nach den Daten der GOG-122-Studie, die sogar die Überlegenheit der Chemotherapie gegenüber der Radiotherapie in den Stadien III und IV hinsichtlich des progressionsfreien Intervalls als auch im Hinblick auf das Gesamtüberleben zeigte, wird derzeit untersucht, inwieweit die Chemotherapie auch in frühen Stadien mit Risikofaktoren zu einer Verbesserung der Prognose führt [4]. Es erfolgt eine kritische Analyse des derzeitigen Standes der adjuvanten wie palliativen Chemotherapie beim Endometriumkarzinom.
Parallel zur Entwicklung einer immer effektiveren tumorspezifischen Therapie ist ein weiterer wesentlicher Aspekt eine möglichst frühe und sichere Diagnosestellung und gleichzeitig die Reduktion der behandlungsbedingten Morbidität. In diesem Zusammenhang wird ein Überblick zur effektiven Primärdiagnostik des Endometriumkarzinoms gegeben und die wichtige Frage des Potentials der Reduktion von Morbidität und Erhalt der Sicherheit durch die laparoskopische Chirurgie evaluiert.
Literatur
1 Amant F, Moermann Ph, Neven P, Timmermann D, Van Limbergen E, Vergote I: Endometrial cancer. Lancet 2005;366:491-505.

2 Shaeffer DT, Randell ME: Adjuvant radiotherapy in endometrial carcinoma. Oncologist 2005; 10:623-631.

3 Martin Hirsch PL, Jarvis G, Kitchener H, Lilford R: Progestagens for endometrial cancer. Cochrane Database Syst Rev 2000;2: CD001040.

4 Kieser K, Oza AM: What's new in systemic therapy for endometrial cancer? Curr Opin Oncol 2005; 17:500-504. 